\title{
BUILDING EFFECTIVE CASE STUDIES FOR MATERIALS ENGINEERING
}

\author{
Vivienne Tam and Marta Cerruti \\ McGill University \\ Vivienne.tam@mail.mcgill.ca
}

\begin{abstract}
Case studies are used to guide students' natural curiosity-driven learning instead of traditional content-heavy lectures. In collaboration with Dr. Marta Cerruti and one other co-teacher, I developed case studies for the undergraduate pre-requisite course "Analytical and Characterization Techniques" (MIME 317) to teach the material characterization concepts such as Atomic Absorption or UV/Vis spectroscopy in case-study driven manner. The process included understanding the professors' desired learning outcomes and finding journal articles that used such concepts to solve real-world problems. Then, I developed handouts to simplify the complicated concepts presented in the articles and crafted questions that students with no background knowledge could still answer given the information provided and the figure/graph from the article. Finally, in delivering the case studies in class, I facilitated group discussion and found that guiding the discussion based on the students' curiosity deepened their understanding of the subject.
\end{abstract}

Keywords: Case study, material characterization, curiosity-driven learning, course development

\section{INTRODUCTION}

While active learning in the classrooms is known to be more effective in deepening a student's grasp of subject knowledge ${ }^{1}$, undergraduate courses in the engineering departments still tend towards a lecturebased approach. As there is a substantial amount of content an instructor is required to cover in each lecture, implementing a more student-driven approach to learning the course material is especially challenging.

With funding from the Gerald W. Farnell Teaching Scholar fellowship, Dr. Marta Cerruti from the Mining and Materials Engineering department at McGill University proposed introducing interrupted case studies in an undergraduate pre-requisite course "Analytical and Characterization Techniques" (MIME 317) as the first pilot test in the materials engineering curriculum. The goal was to see how well this method could be introduced into an existing course, and if

CEEA19; Paper 001

University of Ottawa; June 9-12, 2019 successful, to integrate it into other courses in the materials engineering program and beyond.

MIME 317 is a keystone class in the curriculum, cotaught by four different professors, in which students are exposed to many different characterization techniques including Atomic Absorption or UV-Vis Spectroscopy among others. Due to the number of different concepts covered and the lack of a unifying theme across the various sections of the course, it is difficult for students to connect the dots and make sense of how each of the techniques are useful and in fact, complement each other.

As the graduate student hired to work on developing the case studies that were to be integrated into the curriculum, I was responsible for communicating with each of the professors and crafting case studies that not only guided the students through a discovery process, but also helped them to see the significance of the concepts as they related to each other and then applied to the real world.

The course structure was as follows. There were six main sections of the course: (1) Metallography; (2) Xray generation, diffraction, crystallography; (3) UV-Vis Spectroscopy; (4) Atomic Absorption/Emission Spectroscopy (AAS/AES); (5) Vibrational Spectroscopies (FTIR/Raman); and (6) X-Ray Photoelectron Spectroscopy (XPS). Case studies were developed for (1), (3) - (5).

Each section with a case study began with an introduction session, during which the students had to work through the case study handout in groups. We divided 48 students into 9 groups of 5-6 people each. This handout had to be completed with no background knowledge with the objective of encouraging the students to use their deductive reasoning skills to analyze the figures/graphs and generate hypotheses for the trends observed. The end of the handout concluded with a more complex multi-part question that involved a real-world problem. Students had to work on this question individually outside of class. Over the next few lectures, the professor delivered the course material, referring to the hypotheses and questions that the students came up with while working through the case study. Finally, at the end of the section, the teams were 
given time to share their answers to the multi-part question and discuss the answer they would like to report to the class. With the background knowledge they now had from attending the course lectures, we expected them to have well-founded reasoning for their answers. Then, the teams each shared their answers and the professor led a discussion to conclude the case study for that section.

The students were graded on the following criteria during the last team discussion: team engagement, team focus, team perseverance, quality of response and presentation. This "case study" grade contributed to $10 \%$ of their overall course grade.

In addition to the four topic-based case studies, we developed an introduction and conclusion case study. The introduction case study did not include any of the techniques, but rather was based on the simple concept of differential patterns of bacteria scattering light to detect contamination on chicken tissue. The goal was to ease students into the case study method without having to teach difficult concepts. The conclusion case study, on the other hand, integrated all of the techniques covered in the past case studies. Its goal was to get students to relate the techniques to one another, think about their advantages/disadvantages and thus their complementarity. Finally, in applying the techniques to the real-world problem of paint restoration, we led the students through the process of how one would choose between different techniques depending on the information one has and the desired goal, an extremely important skill for material engineers.

\section{METHODOLOGY}

\subsection{Communication with Professors}

About two months before the beginning of the semester, Dr. Cerruti and I reached out to the other three co-teachers to see who would be interested in integrating the case studies into their section. Two of them responded favorably, however only Dr. Florence Paray who was teaching the first section on metallography was available for an in-person meeting.

Although she was interested in the idea, she was worried about the practicalities of implementing it in the classroom, with questions about whether it would take up too much time, thus requiring that she cut down the material taught. Since I had never taken a course on metallography before, it was a challenge trying to understand the learning outcomes she desired from the case study. However, I took the article she had sent me to read on porosity and fracture behavior in various metal alloys and created three different parts to the

CEEA19; Paper 001

University of Ottawa; June 9-12, 2019 handouts according to the three figures provided in the article. The questions drew on the students' abilities to observe the difference in microstructure and hypothesize on how the solidification time and addition of titanium and strontium contributed to these differences. I also prepared an answer key based on what was written in the journal article. Despite my unfamiliarity with the subject, Dr. Paray was very satisfied with the outcome and how it contributed to an engaging discussion in class.

Preparing the case studies with Dr. Cerruti was more straightforward as having written the Farnell proposal, she already had ideas of what kind of real-world problem could be tackled with each characterization technique. For example, she thought that the spectra of stars could be used to illustrate the basic concepts underlying Atomic Absorption and Emission Spectroscopy. Our first few preliminary meetings were brainstorming sessions as we discussed what concepts were fundamental to communicate for each characterization technique, and what learning outcomes we desired to achieve. For example, Dr. Cerruti told me that she would like the students to learn how to interpret graphs (understanding what the $\mathrm{x}$ and $\mathrm{y}$ axis are measuring) and learn about the importance of error bars. Thus, I made sure to include figure interpretation questions in the handouts. As well, I submitted answers keys with the handouts based on what I believed to be the answer. She would then give me feedback on whether she thought the students were capable of coming to that answer on their own, or if they needed more background information.

\subsection{Case Study Development}

When searching for literature for appropriate articles to use for the case study, I used the following criteria: (1) Availability of clear, comprehensible figures and graphs that were easy to interpret without much additional information; (2) Feasibility of deriving the hypotheses in the article given the figures and some background information; (3) Applicability to an interesting real-world problem and (4) Ability to teach fundamental concepts.

As the topic needed to interest an undergraduate material engineer, I started my literature review with searching for topics I felt would be most relevant and relatable to this demographic. For example, to teach IR and Raman, I looked into food analysis using the spectroscopies as beef tenderness, or food contamination could possibly be detected using these techniques. 
The topics chosen for each technique were as follows: (1) Introduction - Detection of bacteria in chicken breast using laser speckle decorrelation; (2) UV/Vis Spectroscopy - Ratiometric and colorimetric cyanide detection; (3) Atomic Absorption and Emission Spectroscopy - Deciphering the atomic composition of stars; (4) Vibrational spectroscopy - Characterization of edible oils, butters and margarines by Fourier Transform Infrared Spectroscopy and (5) Conclusion Painting Attribution of Restored Art.

When scanning the papers, I mainly assessed the figures provided as a major component of the case studies was the interpretation of figures and graphs. At times, the figures contained too much information and so, I would have to remove information that was irrelevant to the case study in order to not distract the students.

In constructing the handout, I began with a short description of the "real-world problem" to give context and draw attention of the students. The handout was then sub-divided into 3 to 4 parts that began with any relevant (but minimum) background information they had to know to interpret the figure, the figure itself, and subsequent interpretation questions. The handout ended with an application question in which the students had to use the concepts they had learned and either develop their own experiment or method to achieve the outlined objectives.

\subsection{Discussion Facilitation}

During each of the case study sessions, I was present to rotate among the groups and answer any questions they may have had on the handout as well as to gently guide them along the discovery process with probing questions. I was also grading teams on the criteria outlined above and keeping track of time to ensure that we kept to the time we had allotted for each question.

I found that the students were all very engaged and were even asking questions beyond the scope of the handout. As the teams presented their answers, they had to defend the reasoning when their hypotheses differed, causing them to think more deeply about why they would interpret the figure in that particular manner.

\section{RESULTS AND DISCUSSION}

In general, the most difficult part of developing the case studies was understanding the learning outcomes each professor desired to achieve and effectively communicating with them to make sure not only that the case studies achieved these outcomes, but also that the questions were not beyond the capabilities of the students while still challenging them sufficiently.

As this was the first iteration of this method, it worked best with Dr. Cerruti who had a clear idea of what she wanted the case studies to look like. However, in order for this to be effectively implemented in other courses, professors should be able to communicate their vision of what learning objectives the case study should achieve, and potential topic ideas. It is also important for them to be available for feedback on the case studies developed.

In terms of the case study development, choosing an engaging topic with relevance to the students' lives was the most important and also most rewarding part of the process. It helped students make connections between the concepts they learned in class and how they applied to their daily life. The only case study the students had trouble grasping was Case Study \#3 on the atomic composition of stars, and I hypothesize that this was because the topic of this case study was farthest from the students' normal realm of knowledge. Thus, it was hard for them to draw conclusions from the data given and then relate it to the spectroscopic technique.

For discussion facilitation, I highly recommend having the person responsible for developing the course handouts available to rotate between the teams, as questions of clarification regarding the figures/graphs frequently came up. As well, the intentional questioning as we guided the students through the discovery process enhanced their grasp of the subject and pushed them to think in ways they were not used to.

\section{CONCLUSION}

Developing the case studies to teach material characterization techniques involved effective communication with professors concerning learning objectives, a comprehensive literature review looking for papers with clear figures and easily-derived hypotheses and guided team discussion. Rotating between teams during the discussion was helpful in understanding how well students were grasping the concepts and how future handouts should be altered to facilitate their learning. Choosing interesting topics that were relevant to the student's lives was especially beneficial to the case study experience.

\section{REFERENCES}

[1] Bapeler, Paul. Michel. A guide to teaching in the active learning classroom: History, Research and Practice. Stylus Publishing, 2016. 
Proceedings 2019 Canadian Engineering Education Association (CEEA-ACEG19) Conference

CEEA19; Paper 001

University of Ottawa; June 9-12, 2019 